\title{
Fault Localisation of ICs by Lock-in Fluorescent Micro- thermal Imaging (Lock-in FMI)
}

\author{
by F. Altmann*, Th. Riediger*, O. Breitenstein ${ }^{* *}$, J.P. Rakotoniaina** \\ ${ }^{*}$ Fraunhofer Institute for Mechanics of Materials, Halle, Germany, \\ ${ }^{* * M a x}$ Planck Institute of Microstructure Physics, Halle, Germany,
}

\begin{abstract}
An improved method for high resolution surface temperature aquisition using fluorescent microthermal imaging (FMI) is introduced. In combination with lock-inthermographie it can be applied for localisation of leakage currents in microelectronic devices with a precision accuracy of about $1 \mu \mathrm{m}$. As an example hot spot detection of an IC failed with a raised current consumption is demonstrated. The physical failure at the detected hot spot position is verified by TEM cross-section analysis.
\end{abstract}

\section{Introduction}

For quality management and process optimisation the localisation of faulty structures in high-density integrated circuits is an essential assignment. Due to the highly complex circuit structure, defects can not be detected electrically. Defects which cause leakage currents, e.g. gate oxide breakdowns at transistor structures or metallisation shorts, can be detected due to their local heat generation. Up to now this was done essentially by using liquid crystal thermography [1]. A method that is orders of magnitude more sensitive is the IR thermography in lock-in mode (LIT) [2]. An essential drawback of both methods is the limited spatial resolution of about $5 \mu \mathrm{m}$, which is often not enough for precise defect localisation in highest density ICs. An additional thermally detection method is the standard (steady-state) FMI [3] which can be reach high spatial resolutions of less than $1 \mu \mathrm{m}$. However the method has two major disadvantages. At first the sensitivity limit for emitted thermal power is only about $10 \mathrm{~mW}$, which limits the usage to less integrated ICs and power components. Furthermore the images show a strong topographical contrast, which is caused by surface structure effects of the fluorochrome coated sample and mostly dominates over the contrast of the emitted fluorescence light (thermal contrast).

Here a modified FMI technique will be introduced, which is based on lock-in method using a specially developed hardware. It will be shown that this technique essentially lowers the minimum of the detectable heat generation and eliminates the topographical contrast from the taken images. In combination with the lock-in thermography the detection sensitivity and spatial resolution is significantly improved. 


\section{Principle of the standard FMI Process}

Basic element of the FMI is the fluorochrome EuTTA (Europium Tenoyltrifluoroacetonate) which has a strong temperature dependence of the emitted fluorescence radiation in the range of $0 . .60^{\circ} \mathrm{C}$. For stimulation, UV light of $365 \mathrm{~nm}$ wavelength is used, whereas red light of $612 \mathrm{~nm}$ wavelength is emitted. The temperature dependence of the fluorescence is known as quenching effect [4]. Because of this, local heat sources in the fluorescence image going to appear darker compared with the surrounding area. By coating of samples with this fluorochrome it is possible to show thermally emitting failure spots on electrically driven ICs under a light microscope with built-in UV source.

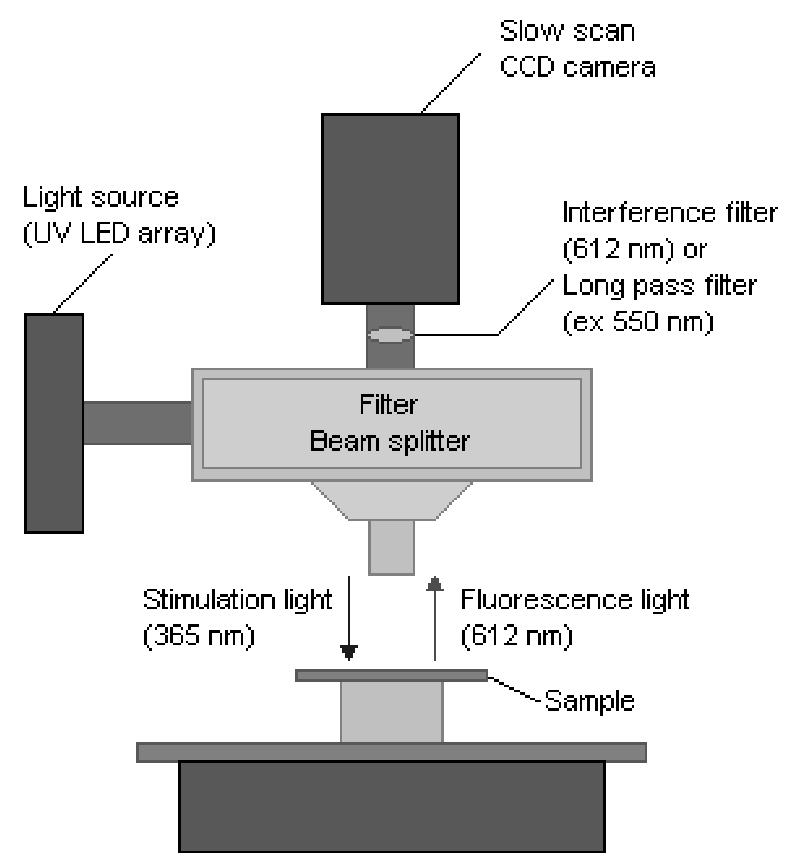

Fig. 1 Assembly for the Fluorescent Microthermal Imaging (FMI)

\section{The Lock-in FMI technique}

Lock-in FMI as much as lock-in thermography means that a periodically pulsed supply voltage (instead of a DC voltage) is applied to the device. The lock-in FMI system developed by us operates with a conventional light microscope and a lownoise, cooled slow scan camera with $1280 \times 1024$ pixels resolution at 256 greyscales. The UV light for the stimulation of the fluorochrome is generated by an compact array of UV diodes with $20 \mathrm{~mW}$ radiated power. Because of the weak fluorescence light, long exposure times up to $1000 \mathrm{~ms}$ are necessary. Due to this fact the 
image exposure time has to be expanded over several lock-in periods. In order to obtain information about the periodic T-modulation, the illuminating UV-LEDs are pulsed at the lock-in frequency. This establishes a heterodyne technique, where the a.c. component of the luminescence signal is mixed down towards d.c.. Hence, the image obtained after exposure over many periods is the sum of a d.c. component, which is independent on the periodic T-modulation, and an a.c. component, which is proportional to the local T-modulation amplitude. This a.c. component also contains the information about the phase difference between the pulsed supply voltage and the pulsed LED illumination, hence this component may be positive or negative. By using a special developed hardware, this phase difference is shifted by $90^{\circ}$ after each taken image, so that after 4 subsequent measurements the images $I^{\circ}, I^{90^{\circ}}, I^{180^{\circ}}$, and $1^{270^{\circ}}$ are representative for these 4 phase positions, see Fig. 2. If necessary, these 4 images may be averaged over subsequent measurements, leading to arbitrarily long effective integration times. From these 4 images the amplitude signal $A$ and the phase signal $\Phi$ are calculated for every pixel position by:

$$
\begin{aligned}
& A(x, y)=\sqrt{\left(I^{0^{\circ}}-I^{180^{\circ}}\right)^{2}+\left(I^{90^{\circ}}-I^{270^{\circ}}\right)^{2}} \\
& \Phi(x, y)=\arctan \left(\frac{I^{90^{\circ}}-I^{270^{\circ}}}{I^{0^{\circ}}-I^{180^{\circ}}}\right)
\end{aligned}
$$

By calculating the differences of always two by $180^{\circ}$ shifted values in (1) and (2), the d.c. part of the fluorescence signal, which is not affected by temperature modulation, completely cancels out. The resulting amplitude image displays the local temperature modulation amplitude, and the resulting phase image essentially describes the time delay of the local temperature modulation referred to the periodic heating. By averaging the result over an appropriate number of lock-in periods the signal-tonoise ratio (SNR) of the lock-in measurement improves proportional to the square root of the acquisition time and may become orders of magnitude better than the SNR of the primary temperature measurement.

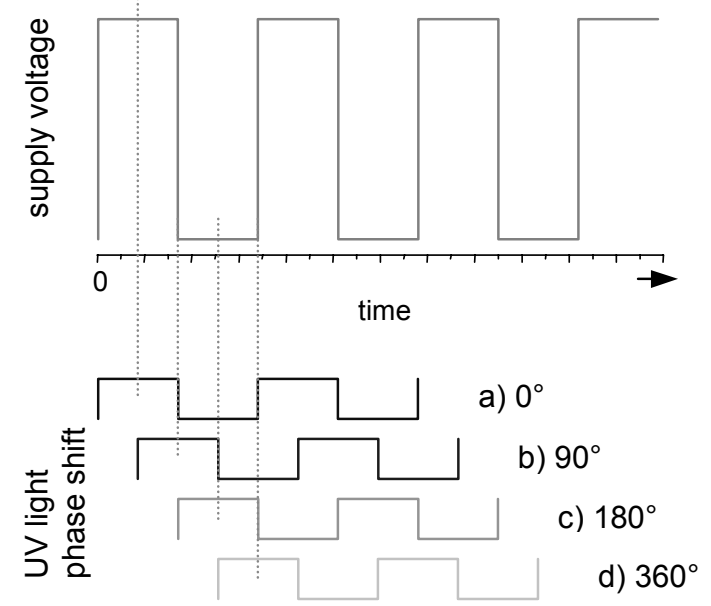

Fig. 2 Phase relations between supply voltage and UV stimulation 


\section{Results}

Figs. 3 an 4 show the result of the hot spot localisation by LIT of an IC failed with a raised current consumption. The overview and high resolution images are overlays of an single IR-emissivity image, showing the IC layout, and the lock-inamplitude image after a few minutes measure time at $5 \mathrm{~V}$ supply voltage an $90 \mu \mathrm{A}$ current flow. The pixel resolution of the high resolution LIT image is about $5 \mu \mathrm{m}$. The LIT-analysis were done using the TDL $384 \mathrm{M}$ 'Lock-in' thermography system by Thermosensorik $\mathrm{GmbH}$ (Erlangen, Germany [5, 6]), which is specialized to the microscopic investigation of electronic components. It is equipped with a Stirlingcooled cadmium-mercury-telluride (CMT) focal plane array (FPA) IR detector head, which is sensitive in the $3 \ldots 5 \mu \mathrm{m}$ wavelength range and at $300 \mathrm{~K}$ object temperature it exhibits a noise level of about $20 \mathrm{mK}$ at a frame rate of $130 \mathrm{~Hz}$. The resolution of this camera is $384 \times 288$ pixel.

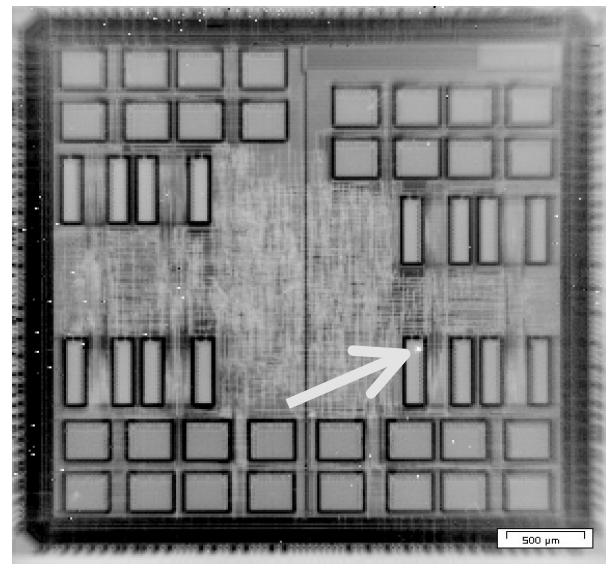

Fig. 3 LIT overview: Hot spot localisation at $3 \mathrm{~Hz}$ lock-in-frequency, $5 \mathrm{~V}$ supply voltage an $90 \mu \mathrm{A}$ current flow

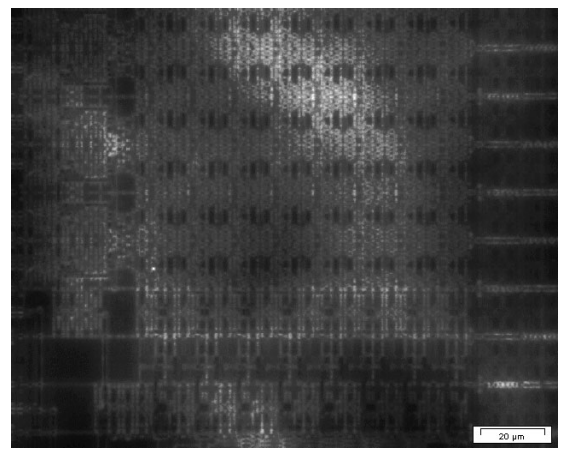

Fig. 5 Fluorescence image (topography, top $(10 \mathrm{~Hz}, 6.5 \mathrm{~V}, 0.3 \mathrm{~mA})$

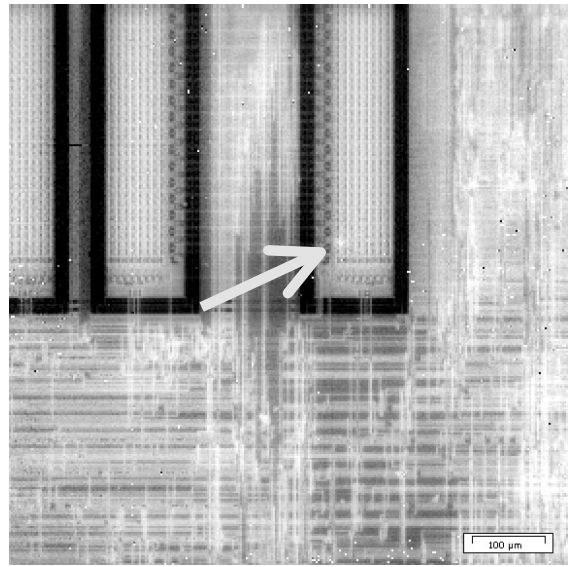

Fig. 4 High resolution LIT: Hot spot localisation by Lock-in thermography (22 Hz, $5 \mathrm{~V}, 90 \mu \mathrm{A}$ )

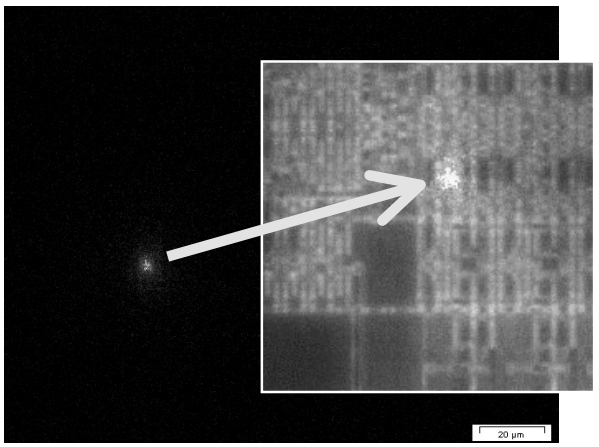

Fig. 6 Lock-in FMI amplitude image (bottom) and correlation of the hot spot to the IC position $(10 \mathrm{~Hz}, 6.5 \mathrm{~V}, 0.3 \mathrm{~mA})$ 
FMl-analysis were done at $6.5 \mathrm{~V}$ supply voltage and $300 \mu \mathrm{A}$ current flow. So the thermal power loss was about $2 \mathrm{~mW}$. Figs. 5 an 6 show in comparison the results of standard FMI an lock-in FMI. After 15 min lock-in FMl-acquisition time at 400x magnification the failure formed a clearly visible spot in the amplitude image. Meanwhile in the standard FMI image the topographical contrast of the chip surface dominates and does not allow to see the weak heat sources.

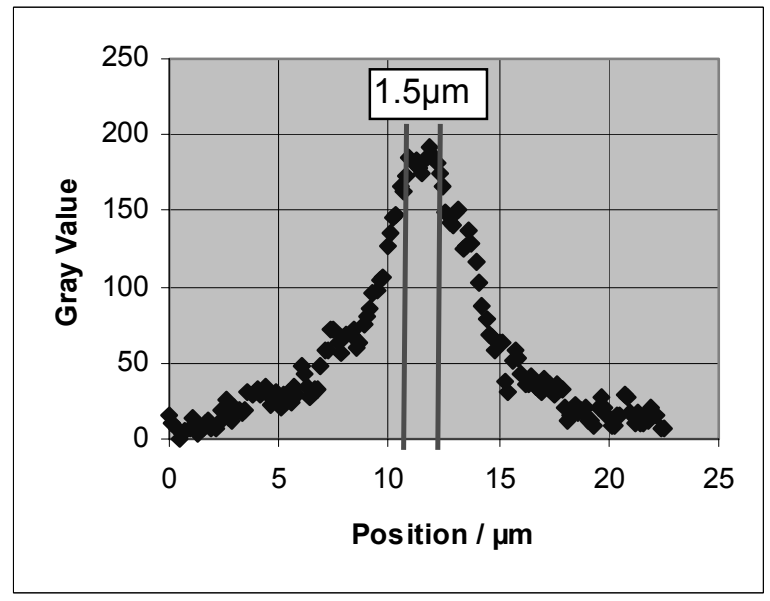

Fig. 7 Linescan over the hot spot, localisation accuracy in this example is $1.5 \mu m$

The pixel resolution of the displayed FMl-images is $0.15 \mu \mathrm{m}$, the optical resolution limit is about twice of this value. It was possible to locate the failure spot with a precision of about $1.5 \mu \mathrm{m}$ at the mentioned measuring parameters, see Fig.7. Indeed the vertical and lateral heat conduction in the IC layer system and Silicon substrate leads to an thermal halo around the local heat sources, which finally limits the reachable lateral resolution. However by a suitable choice of lock-in frequency according the emitted thermal radiation it should be possible to lower the blurring of thermal spots, which is caused by the lateral heat spreading in the Silicon chip material.
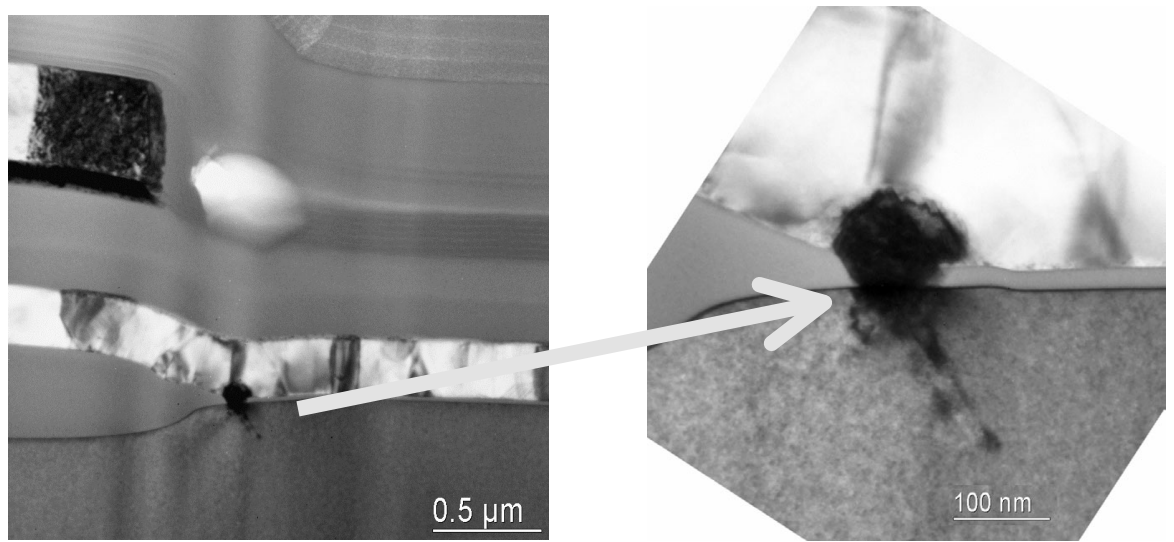

Fig. 8 result of the physical failure analysis after hot spot localisation: TEMcrossection shows an gate oxid breakdown of about 100nm in diameter 
By transmission electron microscopy (TEM) cross-section analysis at the detected hot spot position a gate oxide (GOX) breakdown of a poly Silicon gate were found as root cause of the leakage current, see Fig. 8 . The defect is situated $2 \mu \mathrm{m}$ under the IC surface.

\section{Conclusion}

A new improved FMl method was introduced pushing up the detection limit of local heat sources. In combination with high resolution LIT the lock-in-FMI is very useful for hot spot detection in IC-failure analysis. The lateral resolution of the lock-inFMI is much better than of LIT. In practice the reachable lateral resolution is limited by the layer thickness over heat source and the lateral heat spreading.

\section{REFERENCES}

[1] N. Hirayama, K. Nikawa and M. Nakagiri, Proc. ISTFA 1986, pp. 139-144

[2] O. Breitenstein and M. Langenkamp, Lock-in Thermography - Basics and Use for Functional Diagnostics of Electronic Components, Springer (2003)

[3] P. Kolodner and J.A. Tyson, Appl. Phys. Lett. 40, 782 (1982)

[4] M. L. Bhaumik, Quenching and Temperature Dependence of Fluorescence in Rare-Earth Chelates, The Journal of Chemical Physics 40/12 (1964)

[5] www.thermosensorik.com

[6] O. Breitenstein, J.P. Rakotoniaina, F. Altmann, J. Schulz and G. Linse, Fault Localization and Functional Testing of ICs by Lock-in Thermography, Proc. 28th ISTFA, 29-36 (2002) 